\title{
Antiproteinuric Effect of Chemokine C-C Motif Ligand 2 Inhibition in Subjects with Acute Proliferative Lupus Nephritis
}

\author{
Alessandro Ble $^{a}$ Marta Mosca ${ }^{b}$ Giorgio Di Loreto ${ }^{a} \quad$ Angelo Guglielmotti $^{a}$

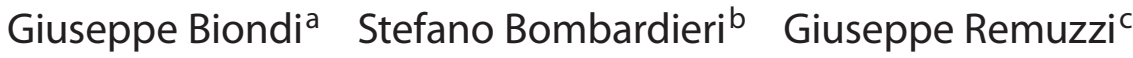 \\ Piero Ruggenentic \\ ${ }^{a}$ Angelini Research Center, Pomezia, ${ }^{b}$ Rheumatology Unit, Department of Internal Medicine, University of Pisa, and \\ 'Mario Negri Institute for Pharmacological Research, Bergamo, Italy
}

\section{Key Words}

Lupus nephritis - Albuminuria • Urinary albumin excretion • Monocyte chemoattractant protein-1 1 Bindarit

\begin{abstract}
Background/Aims: To test the role of chemokine C-C motif ligand 2 (CCL2) in the pathogenesis of lupus nephritis (LN), we evaluated the effects of CCL2 inhibition by bindarit therapy in patients with systemic lupus and active renal disease. Methods: In this proof-of-concept, prospective, randomized, double-blind clinical study, 22 subjects with acute LN were assigned on a 1:1 ratio to 24-week treatment with bindarit $(1,200 \mathrm{mg} /$ day) or matching placebo. All subjects were on the same standardized steroid background therapy. Urinary CCL2, urinary albumin excretion (UAE), estimated glomerular filtration rate, time to remission and time to relapse of LN were compared between groups. Results: Urinary CCL2 significantly decreased during bindarit therapy ( $p=0.008$ vs. baseline) with a reduction that approximated $50 \%$ at study end. CCL2 reduction was paralleled by a persistent reduction in UAE that averaged $80 \%$ vs. baseline and approximated $90 \%$ at study end. Renal function recovery was similar and no difference was found in terms of time to
\end{abstract}

remission and time to relapse of $\mathrm{LN}$ between treatment arms. Treatment was safe and well tolerated in all patients. Conclusion: In lupus subjects with active nephritis, bindarit significantly reduced albuminuria and urinary CCL2 levels. This study provides the background for longer trials to test renoprotective effect of CCL2 inhibition in LN.

Copyright $\odot 2011$ S. Karger AG, Basel

\section{Introduction}

Despite treatment with cytotoxic and immunosuppressive agents, up to $20 \%$ of patients with lupus nephritis (LN), one of the most severe manifestations of systemic lupus erythematosus [1], continues to progress toward renal failure. Patient outcome is further burdened by the severe adverse effects of these drugs [2].

Experimental data suggest that CCL2 (also known as monocyte chemoattractant protein-1, or MCP-1) is upregulated in patients with acute $\mathrm{LN}$ [3-6]. Thus, CCL2

This study was supported by Angelini ACRAF.

\section{KARGER}

Fax +41 613061234 E-Mail karger@karger.ch www.karger.com
(C) 2011 S. Karger AG, Basel

0250-8095/11/0344-0367\$38.00/0

Accessible online at:

www.karger.com/ajn
Angelo Guglielmotti, $\mathrm{PhD}$

Angelini Research Center

Piazzale della Stazione,S. Palomba

IT-00040 Pomezia (Italy)

Tel. +39 069104 5269, E-Mail a.guglielmotti@angelini.it 
upregulation might be a specific target of intervention to prevent or treat this disease.

Chronic treatment with bindarit, a small anti-inflammatory molecule devoid of systemic immunosuppressive effects and activity on arachidonic acid metabolism [7], was associated with CCL2 inhibition in cellular $[8,9]$ and animal [10-14] models. More recent studies, aimed at investigating bindarit's mechanism of action, have demonstrated that the product selectively inhibits mRNA synthesis not only of CCL2 but also of the other members of the monocyte chemotactic protein subfamily of the CC inflammatory chemokines, including CCL7 and CCL8 [9]. The finding that, in addition to blunting renal expression of CCL2, bindarit limited renal damage and improved survival in experimental animals, such as the NZB/W F1 hybrid mice $[13,14]$, with renal features reminiscent of human LN, suggested the possibility of a role for bindarit therapy in human disease. Consistent with this hypothesis, a small uncontrolled open-label study found that an 8-week bindarit treatment [15] reduced urinary albumin and interleukin- 6 excretion in 10 subjects with inactive LN and was well tolerated. These encouraging results, combined with the good safety profile of the compound, paved the way for studies aimed at assessing the risk/benefit profile of bindarit therapy in patients with active disease.

Here we studied the effect of bindarit treatment in a homogeneous cohort of patients with proliferative $\mathrm{LN}$, in the setting of a double-blind, placebo-controlled clinical trial.

\section{Materials and Methods}

\section{Study Design}

This was a prospective, randomized, double-blind, placebocontrolled, proof-of-concept clinical study carried out by 3 Italian Rheumatology Units from February 1997 to September 1999. The study was conducted in accordance with the Declaration of Helsinki. The study protocol was approved by the competent Ethics Committees at each investigational site, and a written informed consent was obtained from all patients before inclusion.

We compared the effect of bindarit and placebo on urinary CCL2 excretion, urinary albumin excretion (UAE), glomerular filtration rate (GFR) as well as on time to remission and/or relapse of LN (see Definitions) in subjects with systemic lupus erythematosus (ARA/ACR [16] criteria) and biopsy-proven class III-IV LN (WHO classification [17]). Subjects with markers of LN activity, including cellular casts or $\geq 10$ red blood cells/field, or recent onset of proteinuria $\geq 1 \mathrm{~g} / 24$ h or proteinuria $\geq 3.5 \mathrm{~g} / 24 \mathrm{~h}$, were eligible for participation in the study. Those with serum creatinine $\geq 1.6 \mathrm{mg} / \mathrm{dl}$ or with severe extra-renal systemic lupus erythematosus manifestations (in the nervous system, heart, vessels, lungs, joints, and/or liver), suggesting the need for immunosuppressive regimens different from that used and standardized for the purpose of the present study, were not included.

\section{Treatments}

Subjects received three 1-gram methylprednisolone intravenous pulses every other day during the 1st week of study, followed by oral methylprednisolone progressively tapered from 40 to $4 \mathrm{mg}$ over 85 days, according to a pre-specified schedule. A 4-mg oral dose of methylprednisolone was maintained as background therapy in both arms up to the end of the 24-week treatment period. After the last dose of intravenous methylprednisolone, at study week 2 , subjects were randomized to bindarit $1,200 \mathrm{mg} /$ day $(2$ tablets each containing $300 \mathrm{mg}$ of bindarit administered orally twice daily) or matching placebo and were prospectively monitored by 13 clinical and laboratory evaluations throughout 25week follow-up.

\section{Definitions}

Complete remission of the LN flare was defined as a reduction in 24-hour urinary protein (24-h UP) to $\leq 0.5 \mathrm{~g}$. Partial remission was defined as either a 50\% reduction in 24-h UP if subjects had baseline 24-h UP values of $<2$ g, or as 24 -h UP concentration $<2 \mathrm{~g}$ in the case of subjects with baseline $24-\mathrm{h}$ UP $\geq 2 \mathrm{~g}$. Provided there was an initial response to treatment, moderate LN relapse was defined as the increment in 24 -h UP concentration to $\geq 2 \mathrm{~g}$, whereas severe relapse was defined as an increase in 24 -h UP concentration to $\geq 3.5 \mathrm{~g}$.

\section{Measurements}

Urinary CCL2 was measured with a validated CCL2 immunoassay (Quantikine Human MCP-1, R\&D System Inc., Minneapolis, Minn., USA). The UP and urinary albumin concentrations were measured in 24-hour collections using a nephelometric assay and an immuno-turbidimetric assay (Tina-quant Albumin, Roche Diagnostics, Indianapolis, Ind., USA), respectively. Serum creatinine was measured using a modified Jaffe's method and GFR was estimated with the Cockcroft-Gault formula [18].

\section{Statistical Analysis}

Differences between treatment groups at baseline were compared using Fisher's exact test for categorical variables and t test or Wilcoxon rank-sum test for continuous variables, as appropriate. Changes in UAE and in urinary CCL2 over time are shown as the relative change (percent change) from baseline. The comparison of change in UAE and CCL2 was initially assessed using arithmetic means, however, since UAE and urinary CCL2 data were not normally distributed (based on the Shapiro-Wilk test for normality), the difference between the 2 treatment groups was examined using a nonparametric equivalent of ANCOVA (rank analysis of covariance), after adjusting for the baseline values and center. The same analysis was applied to change in GFR. The nonparametric Friedman test was used for repeated measures.

Time to event for complete and partial remission and for moderate and severe relapse was explored using Kaplan-Meier curves and comparisons between groups were performed using the logrank test. Missing values were replaced with the last available observations (LOCF method). All differences were evaluated at an 
Table 1. Descriptive statistics of the baseline characteristics of the study sample by treatment group

\begin{tabular}{|c|c|c|c|}
\hline Characteristic & $\begin{array}{l}\text { Bindarit } \\
(\mathrm{n}=11)\end{array}$ & $\begin{array}{l}\text { Placebo } \\
(\mathrm{n}=11)\end{array}$ & $\mathrm{p}$ value \\
\hline Mean age, years (SD) & $31.8(9.3)$ & $35.3(8.6)$ & 0.377 \\
\hline Gender (m:f), $n$ & $0: 11$ & $2: 9$ & 0.476 \\
\hline Caucasian race, $\%$ & 90.9 & 100 & 1.000 \\
\hline In-patient setting, $\%$ & 81.8 & 72.7 & 1.000 \\
\hline Mean height, cm (SD) & $164.9(4.2)$ & $167.2(6.5)$ & 0.341 \\
\hline Median weight, $\mathrm{kg}$ (IR) & $58.7(15.0)$ & $60.0(16.0)$ & 0.870 \\
\hline Mean systolic blood pressure, $\mathrm{mm} \mathrm{Hg}$ (SD) & $128.6(14.5)$ & $137.7(22.0)$ & 0.265 \\
\hline Mean diastolic blood pressure, $\mathrm{mm} \mathrm{Hg}$ (SD) & $81.4(12.3)$ & $85.5(12.9)$ & 0.455 \\
\hline Median serum creatinine, mg/dl (IR) & $0.9(0.4)$ & $1.0(0.4)$ & 0.974 \\
\hline Median estimated GFR, $\mathrm{ml} / \mathrm{min}$ (IR) & $85.6(30.2)$ & $83.0(51.2)$ & 0.860 \\
\hline Anti-native DNA antibodies, $\%$ positive & 90.9 & 44.4 & 0.050 \\
\hline Reduced C3 complement fraction, $\%$ & 81.8 & 72.7 & 1.000 \\
\hline Reduced C4 complement fraction, $\%$ & 63.6 & 63.6 & 1.000 \\
\hline Median 24-hour proteinuria, g (IR) & $1.8(2.6)$ & $3.3(3.2)^{\mathrm{a}}$ & 0.438 \\
\hline Median urinary albumin excretion, $\mu \mathrm{g} / \mathrm{min}$ (IR) & $599.9(1035.0)^{\mathrm{b}}$ & $596.6(307.4)^{\mathrm{a}}$ & 0.733 \\
\hline Median CCL2, pg/ml (IR) & $319.5(587.2)^{\mathrm{b}}$ & $136.0(164.3)^{\mathrm{a}}$ & 0.097 \\
\hline Angiotensin converting enzyme inhibitors, $\%$ & 45.5 & 36.4 & 1.000 \\
\hline$\beta$-Blockers, \% & 18.2 & 0 & 0.476 \\
\hline Calcium channel blockers, $\%$ & 0 & 36.4 & 0.090 \\
\hline Diuretics, \% & 27.3 & 36.4 & 1.000 \\
\hline Statins, \% & 9.1 & 27.3 & 0.587 \\
\hline NSAIDs, \% & 54.5 & 18.2 & 0.183 \\
\hline
\end{tabular}

$\alpha$ level of 0.05 (two-sided) with no corrections for multiplicity due to the exploratory nature of the testing. Data were analyzed with the SAS version 9.1 statistical package.

\section{Results}

Twenty-two subjects (2 men), aged 19-50 years, were randomized: 11 to bindarit and 11 to placebo. All randomized participants were considered in the intentionto-treat analysis with the exception of 1 patient in the placebo group whose baseline urine collection was not collected.

\section{Baseline Characteristics}

All clinical and laboratory characteristics, including UAE and urinary CCL 2 concentrations, as well as the distribution of concomitant treatments, were similar between groups (table 1), with the exception of a trend toward a high proportion of subjects with anti-native DNA antibodies in the bindarit group (90.9 vs. $44.4 \%, \mathrm{p}=0.050$ ).

\section{Urinary CCL2}

As depicted in figure 1, in the bindarit arm, after a transient initial increase, urinary CCL2 decreased from study week 3 up to the end of the study reaching a $49.7 \%$ decrease at study week 25 . On the contrary, in the placebo group, median CCL2 values did not change appreciably throughout the observation period. Consistently, a significant intra-group change in CCL2 over time was observed in the bindarit group (Friedman test, $\mathrm{p}=0.008$ ), but not in the placebo group ( $\mathrm{p}=0.153)$. After adjusting for baseline CCL2 and center, CCL2 levels in the bindarit group were significantly lower $(p<0.05)$ than those in the placebo arm at study weeks 17,19 , and 21 . Changes in urinary CCL2 excretion in individual patients are shown in supplementary material (online supplementary fig. 1; for all online supplementary material, see www. karger.com/doi/10.1159/000330685).

\section{Urinary Albumin Excretion}

As shown in figure 2, in the bindarit group, after a transient initial increase, UAE persistently decreased by 


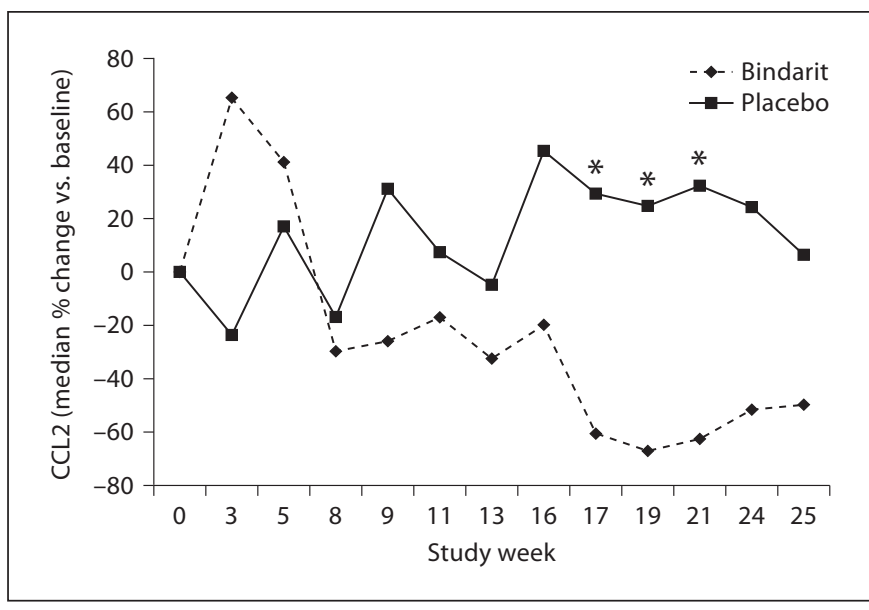

Fig. 1. Relative change (\%) in CCL2 over time compared to baseline. Randomization to bindarit or placebo occurred at study week 1 , after the 1-week i.v. methylprednisolone induction period, such that at week 3 participants had received 2 weeks of experimental treatment. ${ }^{*} \mathrm{p}<0.05$ vs. placebo.

approximately $80 \%$ vs. baseline values, starting from study week 3 ( 2 weeks of treatment) up to the end of the study when UAE reduction peaked to an $87 \%$ decrease by study week 25 , the final week of treatment. In the placebo arm, UAE persistently exceeded the baseline value throughout the whole treatment period (fig. 2). Intragroup changes in UAE vs. baseline approximated statistical significance in the bindarit arm (Friedman test, $\mathrm{p}=$ 0.060 ), whereas no significant change was seen in the placebo arm $(p=0.439)$. After adjusting for baseline UAE and center, changes in UAE were significantly different $(\mathrm{p}<0.05)$ between treatment groups at study weeks 5, 9, 16,24 , and 25. Changes in UAE in individual patients are shown in supplementary material (online suppl. fig. 2).

\section{Other Outcomes}

Estimated GFR did not differ between treatment groups throughout the study.

On follow-up, 3 patients achieved complete remission and 3 partial remission in the bindarit arm compared to 2 and 4 , respectively, in the placebo arm. Of the 6 subjects achieving remission while on bindarit, 2 had a subsequent moderate relapse and 1 had a severe relapse. Of the 6 subjects achieving remission in the placebo arm, 3 had a moderate relapse and 1 had a severe relapse (online suppl. fig. 3). The study was not powered to detect differences in time to remission and time to relapse of $\mathrm{LN}$ between treatment arms.

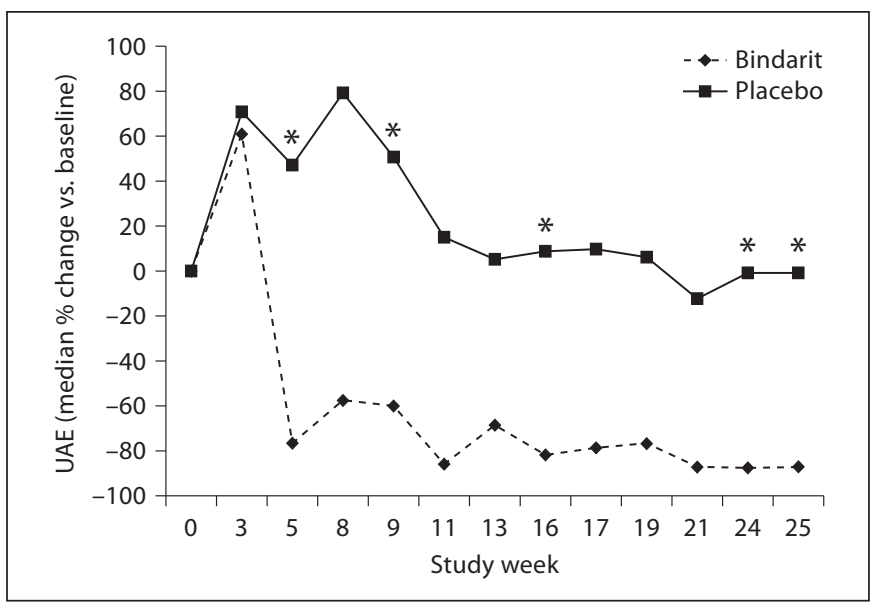

Fig. 2. Relative change (\%) in UAE over time compared to baseline. Randomization to bindarit or placebo occurred at study week 1 , after the 1-week i.v. methylprednisolone induction period, such that at week 3 participants had received 2 weeks of experimental treatment. ${ }^{*} \mathrm{p}<0.05$ vs. placebo.

Double-stranded DNA, C3 and C4 serum levels as well as vital signs including systolic and diastolic blood pressure and heart rate at rest were similar between treatment groups.

\section{Safety}

Treatment was well tolerated in all patients. Overall there were 38 adverse events $(57.6 \%, 4$ serious and 34 nonserious) in the bindarit arm and 28 adverse events (42.4\%, 1 serious and 27 non-serious) in the placebo arm. Only 1 serious adverse event characterized by skin rash with fever and requiring hospitalization was considered to be potentially related to the study treatment.

\section{Discussion}

In this prospective, randomized, double-blind, placebo-controlled proof-of-concept, clinical study we found that 24-week CCL2 inhibition by bindarit therapy was associated with a significant and persistent reduction in UAE compared to placebo in subjects with systemic lupus erythematosus and active nephritis. This is the first evidence of a specific anti-albuminuric effect of bindarit therapy in a placebo-controlled clinical study and our data suggest that this effect could be mediated by the inhibition of CCL2 production in this setting. 
The CCL2-inhibiting activity of bindarit has been previously demonstrated in several preclinical experimental settings, both in vitro $[8,9]$ and in vivo $[10-14]$, but no CCL2-inhibiting effect has been tested or demonstrated in humans so far. Our present findings confirm and extend previous evidence from an uncontrolled study showing that 8-month treatment with $600 \mathrm{mg}$ of bindarit administered orally twice daily significantly reduced UAE in 10 subjects with chronic LN [15]. Here we found that bindarit, as an add-on to gold-standard therapy for active $\mathrm{LN}$, exerted a specific anti-proteinuric effect that was sustained over 24 weeks of chronic therapy.

Mechanisms explaining the anti-albuminuric effect of bindarit in this and other clinical settings is currently a matter of active investigation. It has been shown that CCL2 is upregulated in LN patients [19] and that glomerular podocytes are the predominant cell type overexpressing CCL2 in membranous nephropathies [20]. At the glomerular level, CCL2 is able to activate the migratory capacity of podocytes and to increase their permeability to albumin [21]. Finding that the UAE reduction paralleled the CCL2 reduction in bindarit-treated subjects suggests that in this population the effect of bindarit on UAE could be explained, at least in part, by an inhibitory action on the effects that CCL2 has on podocytes. A similar mechanism has also been suggested in other proteinuric renal diseases, as supported by the evidence that albuminuria is substantially reduced in CCL2 knockout streptozotocin diabetic mice compared to wild-type diabetic control animals [22]. Within the limitation of the small sample size that did not allow drawing any definite conclusion with regard to the considered clinical outcomes, finding that bindarit did not appreciably affect the incidence of disease remissions and recurrences compared to placebo is consistent with previous evidence that bindarit is devoid of specific immunosuppressive effects on acquired immunity [7].

Of note, bindarit therapy was well tolerated throughout the whole study period, with only 1 serious event characterized by skin rash and fever that was possibly related to the study drug. Notably, bindarit did not significantly affect any clinical and laboratory parameter and/or marker of kidney dysfunction over the 24-week treatment period.

\section{Strengths and Limitations}

The major strengths of the present study are the rigorous, prospective and blinded design with a placebo arm, and the careful recording of all considered outcome variables. A limitation of the study is the small number of subjects that however reflects the pilot, explorative nature of the study and the rarity of the considered clinical condition. The study protocol was drafted in 1996 and patients were included from 1997 to 1999, when the benefit of alkylating agents (namely cyclophosphamide) added to steroid therapy were not definitely established. Actually, at that time nephrologists, particularly in Italy, were worried about the severe side effects of these immunosuppressants in particular in young women who represent a substantial proportion of patients affected by LN. This is why the patients included in our present study were not treated with alkylating agents. Thus our present data apply to patients given steroid therapy only and additional studies are needed to assess the effects of CCL2 inhibition therapy on UAE in patients with LN given combined therapy with steroid and alkylating agents.

\section{Conclusions}

The CCL2 inhibitor bindarit added to background steroid therapy was safe and effectively reduced albuminuria in subjects with acute LN. These findings open the perspective for a novel treatment of this disease and may provide the background for further studies aimed at assessing the effect of long-term bindarit therapy on hard clinical outcomes in this population. Moreover, the distinctive mechanism of action suggests the potential benefit of bindarit therapy in other renal diseases associated with albuminuria such as diabetic nephropathy and large part of progressive non-diabetic nephropathies. Studies are needed to assess whether bindarit therapy may be beneficial also in these clinical settings.

\section{Acknowledgements}

We thank Ms. Valeria Di Clemente and Ms. Debora Caucci for their contribution in editing the manuscript.

At the time of the study implementation no web-based, free, publicly accessible database was in use. Studies with bindarit in subjects with LN were authorized by the Italian Ministry of Health (deliberation No. 800.MM/C.35.75/637, November 26, 1991 and No. 88.MM/C.35.75/9465, July 12, 1994).

In July 2009 preliminary results of the study were presented as an oral communication at the 9th Word Conference on Inflammation held in Tokyo, Japan [Inflamm Res 2009;58(suppl 2):S169].

\section{Disclosure Statement}

Alessandro Ble, Giorgio Di Loreto, Angelo Guglielmotti, and Giuseppe Biondi are employees of Angelini ACRAF. 


\section{References}

1 Bagavant H, Fu SM: Pathogenesis of kidney disease in systemic lupus erythematosus. Curr Opin Reumatol 2009;21:489-494.

$\checkmark 2$ Singh S, Saxena R: Lupus nephritis. Am J Med Sci 2009;337:451-460.

$\checkmark 3$ Vielhauer V, Anders HJ, Schlöndorff D: Chemokines and chemokine receptors as therapeutic targets in lupus nephritis. Semin Nephrol 2007;27:81-97.

$\checkmark 4$ Noris M, Bernasconi S, Casiraghi F, Sozzani S, Gotti E, Remuzzi G, Mantovani A: Monocyte chemoattractant protein-1 is excreted in excessive amounts in the urine of patients with lupus nephritis. Lab Invest 1995;73: 804-809.

5 Rovin BH, Song H, Birmingham DJ, Hebert LA, Yu CY, Nagaraja HN: Urine chemokines as biomarkers of human systemic lupus erythematosus activity. J Am Soc Nephrol 2005: 16:467-73.

6 Marks SD, Williams SJ, Tullus K, Sebire NJ: Glomerular expression of monocyte chemoattractant protein-1 is predictive of poor renal prognosis in pediatric lupus nephritis. Nephrol Dial Transplant 2008;23:35213526.

-7 Cioli V, Ciarniello MG, Guglielmotti A, Luparini MR, Durando L, Martinelli B, Catanese B, Fava L, Silvestrini B: A new protein antidenaturant agent, bindarit, reduces secondary phase of adjuvant arthritis in rats. J Rheumatol 1992:19:1735-1742.

$>_{8}$ Sironi M, Guglielmotti A, Polentarutti N, Fioretti F, Milanese C, Romano M, Vigini C, Coletta I, Sozzani S, Bernasconi S, Vecchi A, Pinza M, Mantovani A: A small synthetic molecule capable of preferentially inhibiting the production of the $\mathrm{CC}$ chemokine monocyte chemotactic protein-1. Eur Cytokine Netw 1999; 10:437-442.
9 Mirolo M, Fabbri M, Sironi M, Vecchi A, Guglielmotti A, Mangano G, Biondi G, Locati M, Mantovani A: Impact of the anti-inflammatory agent bindarit on the chemokinome: selective inhibition of the monocyte chemotactic proteins. Eur Cytokine Netw 2008;19:119-122.

10 Guglielmotti A, D’Onofrio E, Coletta I, Aquilini L, Milanese C, Pinza M: Amelioration of rat adjuvant arthritis by therapeutic treatment with bindarit, an inhibitor of CCL2/MCP-1 and TNF-alpha production. Inflamm Res 2002;51:252-258.

11 Bhatia M, Ramnath RD, Chevali L, Guglielmotti A: Treatment with bindarit, a blocker of CCL2/MCP-1 synthesis, protects mice against acute pancreatitis. Am J Physiol Gastrointest Liver Physiol 2005;288:G12591265.

12 Bhatia M, Landolfi C, Basta F, Bovi G, Ramnath RD, de Joannon AC, Guglielmotti: Treatment with bindarit, an inhibitor of CCL2/MCP-1 synthesis, protects mice against trinitrobenzene sulfonic acid-induced colitis. Inflamm Res 2008;57:464471.

$\checkmark 13$ Zoja C, Corna D, Benedetti G, Morigi M, Donadelli R, Guglielmotti A, Pinza M, Bertani T, Remuzzi G: Bindarit retards renal disease and prolongs survival in murine lupus autoimmune disease. Kidney Int 1998;53: 726-734.

14 Guglielmotti A, Aquilini L, D’Onofrio E, Rosignoli MT, Milanese C, Pinza M: Bindarit prolongs survival and reduces renal damage in NZB/W lupus mice. Clin Exp Rheumatol 1998;16:149-154.

15 Viganò G, Gotti E, Casiraghi F, Noris M, Taddei I, Dionisio P, Remuzzi G: Bindarit reduces urinary albumin excretion (UAE) and urinary interleukin-6 (IL-6) in patients with proliferative lupus nephritis. Proc 28th Annu Meeting Am Soc Nephrol. J Am Soc Nephrol 1995;6:434.
16 Tan EM, Cohen AS, Fries JF, Masi AT, McShane DJ, Rothfield NF, Schaller JG, Talal N, Winchester RJ: The 1982 revised criteria for the classification of systemic lupus erythematosus. Arthritis Rheum 1982;25:12711277.

17 McCluskey RT: Lupus nephritis; in Sommers SC (ed): Kidney Pathology Decennial 19661975. East Norwalk, Appleton-CenturyCrofts, 1975, pp 435-450.

18 Cockcroft DW, Gault MH: Prediction of creatinine clearance from serum creatinine. Nephron 1976;16:31-41.

19 Noris M, Bernasconi S, Casiraghi F, Sozzani S, Gotti E, Remuzzi G, Mantovani A: Monocyte chemoattractant protein-1 is excreted in excessive amounts in the urine of patients with lupus nephritis. Lab Invest 1995;73: 804-809.

20 Tarabra E, Giunti S, Barutta F, Salvidio G, Burt D, Deferrari G, Gambino R, Vergola D, Pinach S, Perin PC, Camussi G, Gruden G: Effect of the monocyte chemoattractant protein-1/CC chemokine receptor 2 system on nephrin expression in streptozotocin-treated mice and human cultured podocytes. Diabetes 2009;58:2109-2118.

21 Lee EY, Chung CH, Khoury CC, Yeo TK, Pyagay PE, Wang A, Chen S: The monocyte chemoattractant protein-1/CCR2 loop, inducible by TGF-beta, increases podocyte motilityand albumin permeability. Am J Physiol Renal Physiol 2009;297:F85-F94.

22 Chow FY, Nikolic-Paterson DJ, Ozols E, Atkins RC, Rollin BJ, Tesch GH: Monocyte chemoattractant protein-1 promotes the development of diabetic renal injury in streptozotocin-treated mice. Kidney Int 2006;69: $73-80$. 